\title{
Circulation of an object immersed in a bubbling fluidized bed
}

\author{
A. Soria-Verdugo*, L.M. Garcia-Gutierrez, S. Sanchez-Delgado, U. Ruiz-Rivas

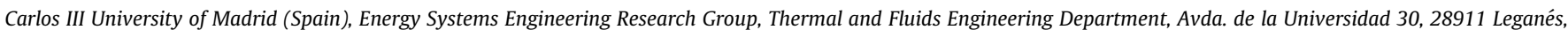 \\ Madrid, Spain
}

Keywords:

Gas fluidization

Bubbling bed

Object motion

Object jump

Sinking and rising velocity

Circulation time

\begin{abstract}
A B S T R A C T
The motion of a large object in a bubbling fluidized bed was experimentally studied using digital image analysis (DIA). The experiments were performed in a 2 D bubbling fluidized bed with glass spheres as bed material. The object motion was measured using non intrusive tracking techniques, while independent measurements of the dense phase velocity (using Particle Image Velocimetry (PIV)) and bubble velocity (using DIA) were carried out. The effect of the dimensionless gas velocity on the object motion was also analyzed.

This work characterizes the circulation patterns of an object with a density similar to that of the bed, but much larger in size. Object size and density remained constant throughout the experiments. A comparison between the motion of sinking objects and the motion of the dense phase provided evidence of the feeble effect of buoyant forces on the motion of sinking objects. In contrast, the motion of rising objects is linked to the motion of bubbles. It was found that objects may be raised to the surface of the bed either by the action of a single bubble (one jump) or by several passing bubbles (multiple jumps). Based on these results, the circulation time of objects throughout the bed is a function of two parameters: the maximum depth attained by an object and the number of jumps during its rising path. This relationship is presented along and the multiple jumps phenomenon is studied in detail. Finally, an estimate of the circulation time of an object based on semi empirical expressions is presented for different dimensionless gas velocities. The probability density function of the circulation time shows two different modes as the object was less prone to be raised at moderate depths. The estimate of the circulation time was found to be in good agreement with our experimental data.
\end{abstract}

\section{Introduction}

Bubbling fluidized beds (BFB) are widely used in the industry, from pharmaceutical to energy conversion systems; they are well suited for processes involving heat and mass transfer as well as chemical reactions. Examples include drying processes, thermal conversion of solid fuels, and coating of particles. A wide range of these applications involves the motion of large objects within the bed. Examples of objects commonly used in a BFB include fuel particles, catalysts, reactants, and agglomerates; these objects will sink with the dense phase and rise in (or in a trail of) the bubbles. Buoyancy effects may play a significant role in the motion of objects in BFB, depending on the density ratio between the object and the bed. Object motion patterns and their ability to move throughout the bed or stay in a restricted zone has a paramount effect on several factors, including the performance of the reactor, the reaction time, the existence of hot or cold spots, and the behavior of agglomerates.

\footnotetext{
* Corresponding author. Tel.: +34 91 6248884; fax: +34 916249430 .

E-mail address: asoria@ing.uc3m.es (A. Soria-Verdugo).
}

Several studies have focused on the motion of an object within a $\mathrm{BFB}$, and most of these studies are based on experimental analysis, including experiments in $2 \mathrm{D}$ and $3 \mathrm{D}$ beds and using different measurement techniques. Attending the object characteristics, the studies can be divided into two categories: those where the object had a similar density and size to the bed material, as well as those where the object was larger. The former were intended to characterize the motion of the dense phase, while the latter provided information of the motion of a fuel, catalyst or agglom erate particle. Several studies have investigated the motion of tracing particles that follow the dense phase, using tracking and non tracking techniques. Tomography tracking techniques that are employed to characterize the motion of objects in $3 \mathrm{D}$ beds include positron emission (Stein et al., 2000), X ray (Grassler and Wirth, 2000), and electrical capacitance (Du et al., 2002) methods, while optical techniques (Shen et al., 2004; Bokkers et al., 2004) are used in $2 \mathrm{D}$ beds. The motion of objects in fluidized beds has also been analyzed using non tracking techniques (collecting data of the motion at a particular position in the bed), such as the Laser Doppler Anemometry (Ibsen et al., 2002).

The first studies with regard to the motion of large objects were reviewed by Kunii and Levenspiel (1991). Rios et al. (1986) studied 
the motion of large objects in $2 \mathrm{D}$ and $3 \mathrm{D}$ beds and discussed the sinking and rising processes. They observed that an object was not always raised to the surface of the bed by a single bubble, but was rather lifted small distances by a succession of passing bubbles, rising in a series of small jumps. Pallarès and Johnsson (2006) analyzed the motion of a phosphorescent particle in a $2 \mathrm{D}$ bed for several configurations and observed that a characteristic 2 vortex configura tion appears in most cases, with the exception of those where the bed was extremely shallow. In a 2 vortex configuration, an object with a similar density to the dense phase sinks at the side of the bed and rises at the center. Several authors have studied the sinking process. Lim and Agarwal (1994) found that the sinking velocity of a large object in a $2 \mathrm{D}$ bed was in good agreement with the Kunii and Levenspiel correlation for the velocity of the dense phase, for density ratios around 1. However, Hoffmann et al. (1993) showed that for large density ratios, a relative motion between an object and the dense phase existed and was linear with density. Nguyen and Grace (1978) and Rees et al. (2005) stated that the net buoyant force on an object in a fluidized bed might differ from what would be expected based on its density, due to a de fluidized hood of bed material that appears on top of the object. The rising velocity is related to the bubble velocity in a complex way. Complicating factors include the multiple jumps effect observed by Rios et al. (1986) and the relative motion between bubble and object, which are far more important than those between object and dense phase in the sinking process. The experimental works of Nienow et al. (1978), Lim and Agarwal (1994), and more recently Rees et al. (2005) showed different results for the mean rising velocity, which is roughly between $10 \%$ and $30 \%$ of the mean bubble velocity along the bed.

There is a continued need to investigate the fundamentals of the motion of an object immersed in a fluidized bed, including the study of the limits of proper circulation, the description of the circulation patterns and preferential paths, the evaluation of the circulation time, and the study of the incidence of buoyant forces. This work evaluates the motion of a large object in a $0.5 \times 0.5 \mathrm{~m}^{2} 2 \mathrm{D}$ fluidized bed with a similar density to that of the bed. The object size and density remained constant during each experiment. The general patterns are presented and object sinking and rising velocities are determined. The focus of this study was to characterize the cycles described by the object, sinking from the surface of the bed and rising back to this point. The circulation time, which is the time spent by the object in a cycle, was measured, and its dependence on the number of jumps needed and the maximum depth attained was analyzed. The effect of the dimensionless gas velocity on circulation time of the object was also analyzed. Finally, a simple estimate of the circulation time of the object based on semi empirical expressions was made.

\section{Experimental setup}

The experiments were carried out in a $2 \mathrm{D}$ column with a width $(W)$ of $0.5 \mathrm{~m}$, a height $(H)$ of $2 \mathrm{~m}$, and a thickness $(T)$ of $0.01 \mathrm{~m}$. The column was made of aluminum with a glass front wall to allow an optical access. The bed material was glass spheres, ballotini particles, with a diameter of $600800 \mu \mathrm{m}$, and a skeletal density of $2500 \mathrm{~kg} / \mathrm{m}^{3}$, type B according to Geldart's classification (Geldart, 1973). The bulk density of the fixed bed was measured to be $1560 \mathrm{~kg} / \mathrm{m}^{3}$. Three piezo electric pressure transducers (Kistler type 5015) were used to measure pressure fluctuations in the bed at the plenum chamber, at 0.2 and $0.3 \mathrm{~m}$ above the distributor. The pressure signals were analyzed in the time and in the frequency domains to obtain the minimum fluidization velocity $\left(U_{m f}, 0.32 \mathrm{~m} / \mathrm{s}\right)$ and the characteristic frequency of the bed $\left(f_{B}, 1.51 \mathrm{~Hz}\right)$, respectively. The fixed bed height $\left(h_{B}\right)$ was $0.5 \mathrm{~m}$, which is high enough to guarantee the existence of a preferential path of bubbles, operating in a 2 vortex configuration according to Pallarès and Johnsson (2006) nomenclature. The dimensionless gas velocity $\left(U / U_{m f}\right)$ was varied between 1.5 and 3 (2.5 being the nominal case), corresponding to a bubbling fluidization regime and reaching gas velocities similar to that employed in industrial applications (Koornneef et al., 2007). Higher dimensionless gas velocities pro duced a slugging regime, which is not the focus of this work. A schematic diagram of the experimental facility is presented in Fig. 1.

The air distributor was a perforated plate made of aluminum $\left(0.5 \times 0.01 \mathrm{~m}^{2}\right)$ with 2 lines of holes, each consisting of 50 holes of $0.001 \mathrm{~m}$ diameter $\left(d_{0}\right)$ and spaced $0.01 \mathrm{~m}$ apart $\left(d_{1}\right)$. The two lines were separated by a distance $(s)$ of $0.0033 \mathrm{~m}$ (see Fig. 2a). For all experiments in this publication, the pressure drop through the distributor (Fig. 2b) was high enough $\left(\Delta P_{D}>0.3 \Delta P_{B}\right)$ to ensure that the bed and the air supply system were independent (Karri and Werther, 2003; Johnsson et al., 2000). Some industrial devices may operate with a smaller pressure drop in the distributor and this may alter the motion of objects within the bed. A typical drawback in such cases is the incidence of de fluidized zones. Then, the motion of the object should be studied in two cases, depending whether it reaches a de fluidized zone or not. In our study, this fact was not considered.

The object was a cylindrical biomass pellet with a density of $1508 \mathrm{~kg} / \mathrm{m}^{3}$, a diameter of $0.0064 \mathrm{~m}$, and a length of $0.0192 \mathrm{~m}$. Therefore, the object fixed bed density ratio was 0.97 , and the object bed material characteristic length ratio was approximately 9. The object density was selected in order to reduce the effect of buoyant forces. The effect of buoyant forces will be the focus of future work.

Three different acquisition systems were used during the experi ments. A standard camera (Nikon D80 10.6 Mpx) was used to obtain the average motion patterns of the object and its velocity. The acquisition frequency was 1.6 fps for position measurements (motion patterns) and $30 \mathrm{fps}$ for velocity measurements. The dense phase and bubble characterizations were obtained using a high speed video camera (Redlake Motion pro X3 $4 \mathrm{~GB}$ ) with an acquisition frequency of $125 \mathrm{fps}$. Average measurements of the object's position and velocity

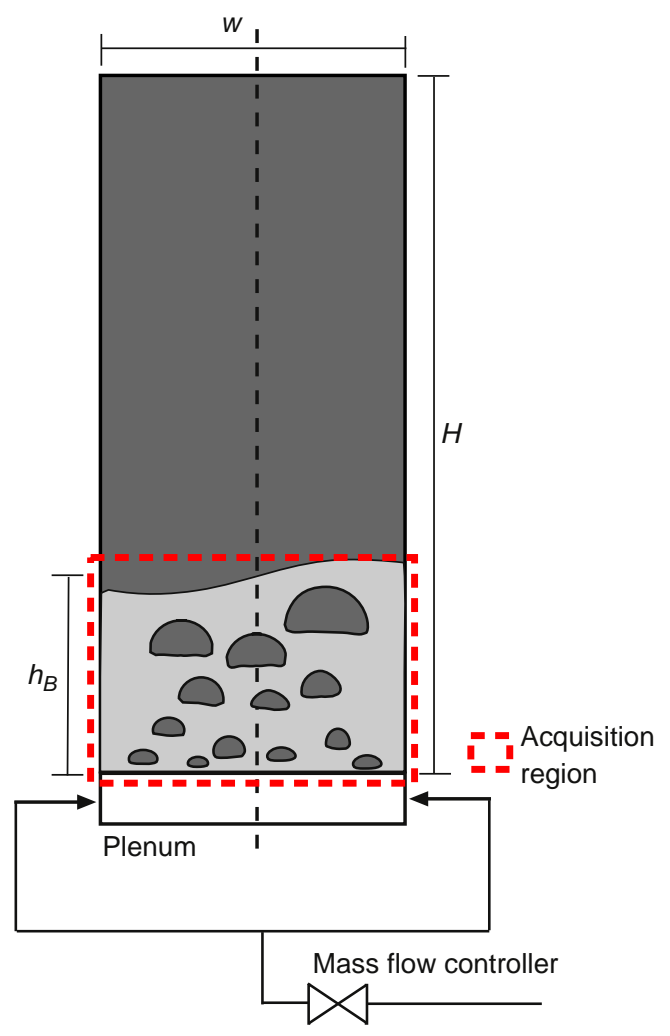

Fig. 1. Scheme of experimental facility. 
and dense phase velocities were obtained. Dense phase and bubbles were discriminated using a threshold of the grayscale map. For the PIV measurements of the dense phase velocity, the MatLab ${ }^{\circledR}$ MatPIV 1.6.1 was used (Sveen, 2004). Digital Image Analysis and averaging techniques were also performed using MatLab ${ }^{\circledR}$.

The measurements of object motion (both position and velocity) were carried out in complete darkness. The object was coated with strontium aluminate to emit green light. Averaged object position maps were obtained from 16,000 images, representing $10,000 \mathrm{~s}$ of BFB activity. For object velocity, 21,600 images were recorded, representing $720 \mathrm{~s}$. Four spotlights of $650 \mathrm{~W}$ were used during the characterization of the bubbles and dense phase to get a uniform illumination on the bed; the rear wall of the bed was covered by a black paper to obtain a high contrast between dense phase (white) and bubbles (black). Bubble and dense phase velocities were determined by averaging 9810 images, which represent $78 \mathrm{~s}$ of

a

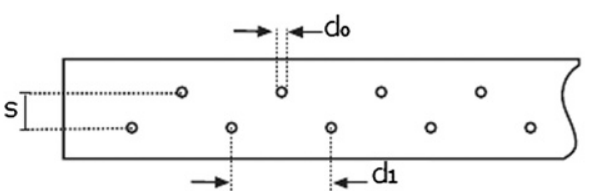

b

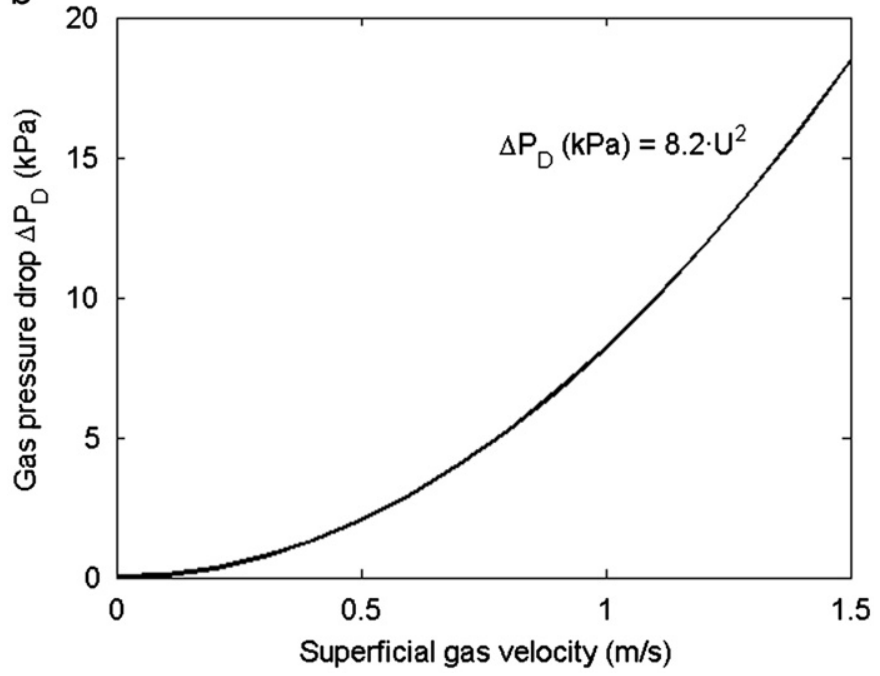

Fig. 2. (a) Distributor design and (b) pressure drop through the distributor.
BFB activity. This measurement time (78 s), although rather small, has proved to give reproducible results.

\section{Results}

As explained in Section 1, it is generally accepted that objects with densities similar to that of the bed will describe sinking rising cycles throughout the whole bed, where the sinking motion is similar to that of the dense phase, and the rising motion is composed of a number of sudden jumps, as a result of the raising effect of passing bubbles. Experimental results in this section include the characterization of the dense phase and bubble motion, the averaged motion patterns of objects, and the study of object average sinking and rising velocities.

\subsection{Dense phase and bubbles}

Two different post processing techniques, digital image analysis (DIA) and Particle Image Velocimetry (PIV), were applied to char acterize the dense phase and bubbles behavior throughout the bed. DIA was applied to establish the boundaries between the dense and the bubble phases. The analysis was based on the calculation and application of a threshold level over each image (Otsu, 1979). Using this technique, the two phases (dense and bubble phases) were clearly identified. Furthermore, different bubble properties could be calculated, such as the equivalent diameter and the mass center of the bubble. MatPIV 1.6.1 (Sveen, 2004) was employed to calculate PIV in the bed. Several authors have used this application on $2 \mathrm{D}$ fluidized beds to calculate and analyze dense phase velocities (Müller et al. 2007 and Almendros Ibáñez et al., 2009) and their fluctuations (Sanchez Delgado et al., 2010). Interrogation windows of $16 \times 16$ pixels with a $50 \%$ overlap were used in the PIV analyses.

The application of the threshold level to the grayscale image converts it into a binary image, where the pixels occupied by bubbles have a value of $C=0$, while the pixels occupied by the dense phase have a value $C=1$. Thus, the relative frequency that a point is occupied by the dense phase, $C$, can be calculated as follows:

$C(i, j)=\frac{\sum_{n}^{N_{P}} C_{1}(i, j)}{N_{P}}$

where $C_{n}$ is the instantaneous concentration once the threshold is applied ( 1 represents the dense phase and 0 represents the bubble phase), $N_{P}$ is the total number of images, and $i$ and $j$ represent the pixel $x$ and $y$ positions, respectively.
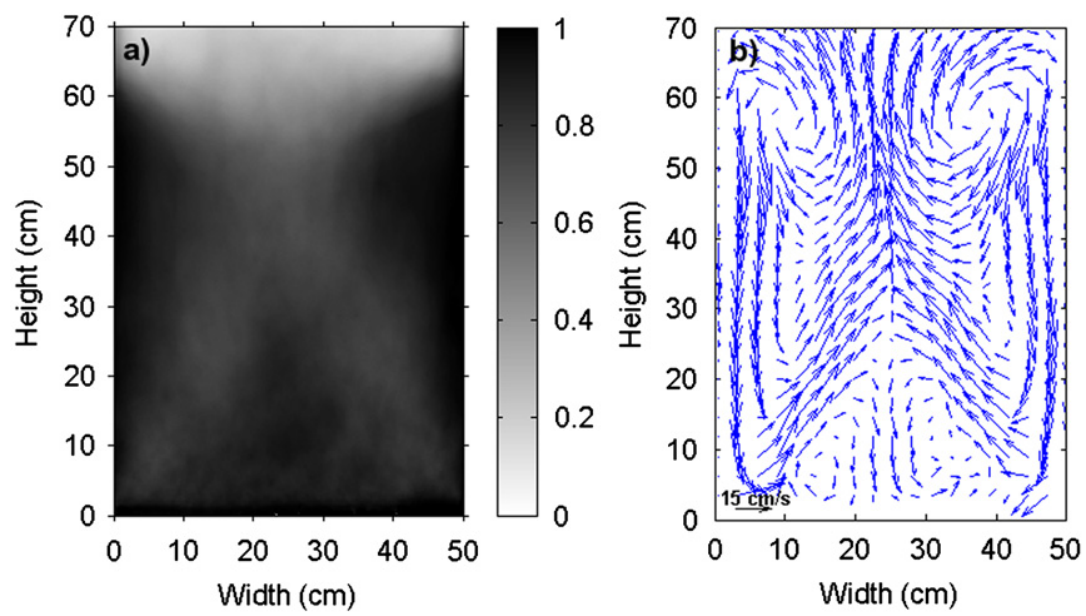

Fig. 3. (a) Relative frequency of dense phase and (b) time-averaged dense phase velocity. 
The regions where $C(i, j)$ reaches higher values, close to one, represent a lower relative frequency of bubbles, and the regions with lower values of $C(i, j)$ represent the preferential bubble paths. Fig. 3a shows the relative frequency of the dense phase at each location of the bed, $C(x, y)$. In the lower zone of the bed, close to the distributor, the relative frequency is almost uniform, so bubbles are equally distributed through the width of the bed in this zone. Then, two symmetrical preferential bubble paths are formed and developed from the distributor corners to the center of the bed. Further away from the distributor, the dense phase is more concentrated at the sides of the bed, while bubbles appear at the middle due to coalescence.

The time averaged dense phase velocity field (Fig. 3b) was calculated by averaging the velocities obtained by PIV, as described in Laverman et al. (2008). The dense phase transport phenomena induced by bubbles during the rising motion (Rowe, 1973; Sanchez Delgado et al., 2010) is described in Fig. 3b. The dense phase was found to descend at the sides of the bed while the ascending motion was located in the middle, corresponding to the bubbles' preferential path. Note that there was a triangular region of low velocities in the bottom zone, where small bubbles with no distinct preferential paths were present.

\subsection{Object motion patterns}

Previous studies (Lim and Agarwal, 1994) showed that objects that differ in size compared to the bed material circulate throughout the whole bed for a wide range of object densities around that of the fluidized bed. In contrast, much denser objects (jetsam) fall straight to the bottom and remain there, while much lighter objects (floatsam) are permanently kept near the surface of the bed. In this sense, a direct measurement of the relative frequency of the object present at each position is important to characterize an object circulation.

Sixteen thousand images showing the object emitting a green light were transformed into black and white images applying a threshold. In these images, the center of mass of the object was determined, and the relative frequency of objects at certain positions of the bed was calculated, dividing it in 280 cells of $0.05 \times 0.05 \mathrm{~m}^{2}$. The relative frequency at each cell was calculated to be the number of times that the center of mass of the object was present at that cell divided by the total number of images. There fore, in zones where objects move with a lower velocity, a higher relative frequency will result. Fig. 4 shows the relative frequency of objects at each position of the bed.

A comparison between Figs. 3 and 4 shows a similar behavior for both the object and the dense phase. An interesting difference is found at the central bottom zone, where objects seldom access. For a clearer understanding of object paths, a distinction between rising and sinking paths can be made in the calculations. Fig. 5 shows the relative frequency of rising and sinking objects as a function of bed width, considering the entire height of the bed.

The preferential paths for the sinking and rising motions are evident in Fig. 5. The objects rise at the center of the bed by the action of bubbles and sink at the sides, following the dense phase. This is in accordance with bubble patterns and preferential paths in a 2 D BFB shown in Fig. 3. This behavior is also in agreement with the results of Pallarès and Johnsson (2006).

The relative frequency of objects at each the $y$ position (height above the distributor) is of paramount importance to characterize an object circulation, since it is an indicator of the capability of producing a homogeneous axial mixing. Fig. 6a shows the relative frequency to find the object at each height.

Fig. 6a shows the distribution of the object in the upper $2 / 3$ of the bed was fairly homogeneous, while in the region near the

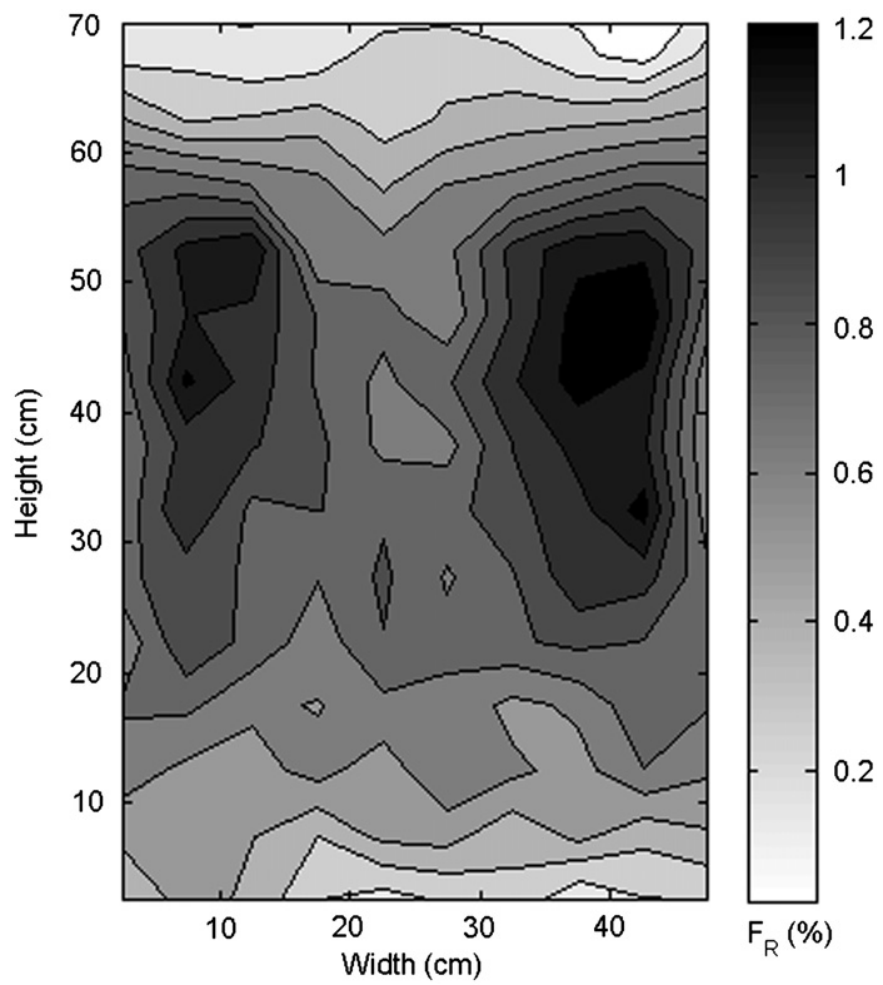

Fig. 4. Relative frequency of objects at a certain position in the bed. Nominal case $\left(U / U_{m f}\right.$ 2.5).

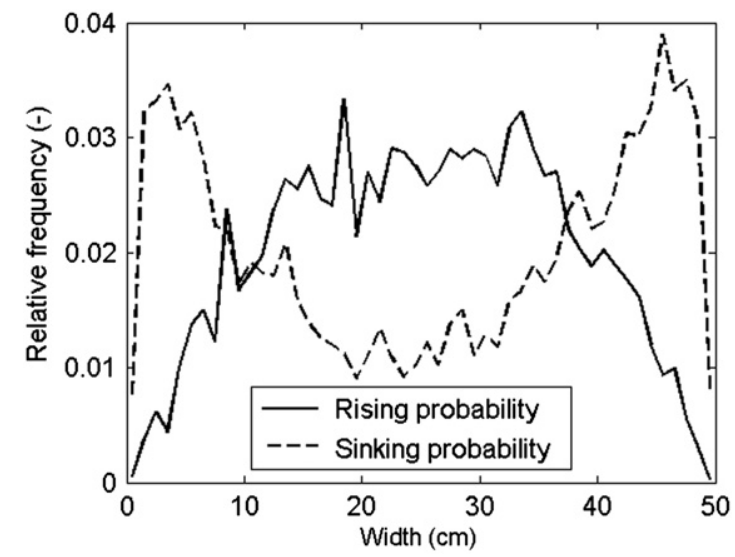

Fig. 5. Relative frequency of a rising or a sinking object as a function of the $x$-coordinate of the bed (width). Nominal case $\left(U / U_{m f} \quad 2.5\right)$.

distributor, at heights lower than $0.2 \mathrm{~m}$, objects were seldom present. This may be related to the inability of objects to reach the central bottom region of the bed described earlier (comparing Figs. 3a and 4).

To analyze the effect of the dimensionless gas velocity on an object distribution through the entire height of the bed, the relative frequency to find the object in the upper part of the bed (higher than $0.3 \mathrm{~m}$, considering the fluidized bed average height to be $0.6 \mathrm{~m}, 1.2$ times the fixed bed height) was calculated. This para meter would be close to $50 \%$ for a homogeneous distribution of the object in the bed, close to $100 \%$ for floatsam objects that keep near the surface of the bed, and close to $0 \%$ for jetsam objects which are always deep in the bed and seldom appear on the freeboard. The effect of the dimensionless gas velocity on this parameter is analyzed in Fig. 6b, where the relative frequency to find the object in the upper half of the bed is plotted. For a range of 

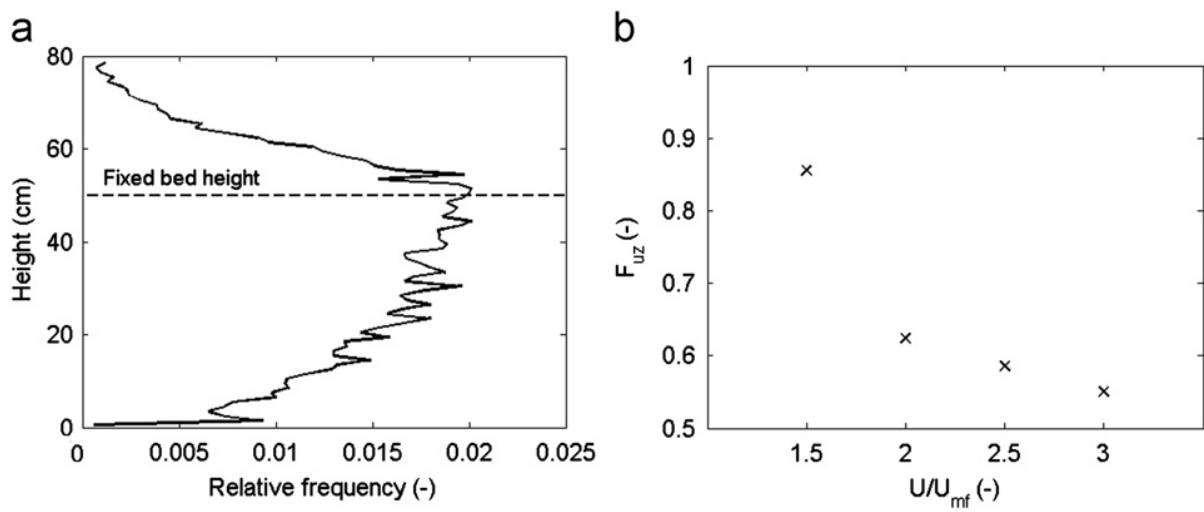

Fig. 6. (a) Relative frequency to find the object at a determined position as a function of the $y$-coordinate (height from the distributor) for the nominal case $\left(U / U_{m f} 2.5\right)$ and (b) effect of the dimensionless gas velocity on the distribution of the object through the bed.
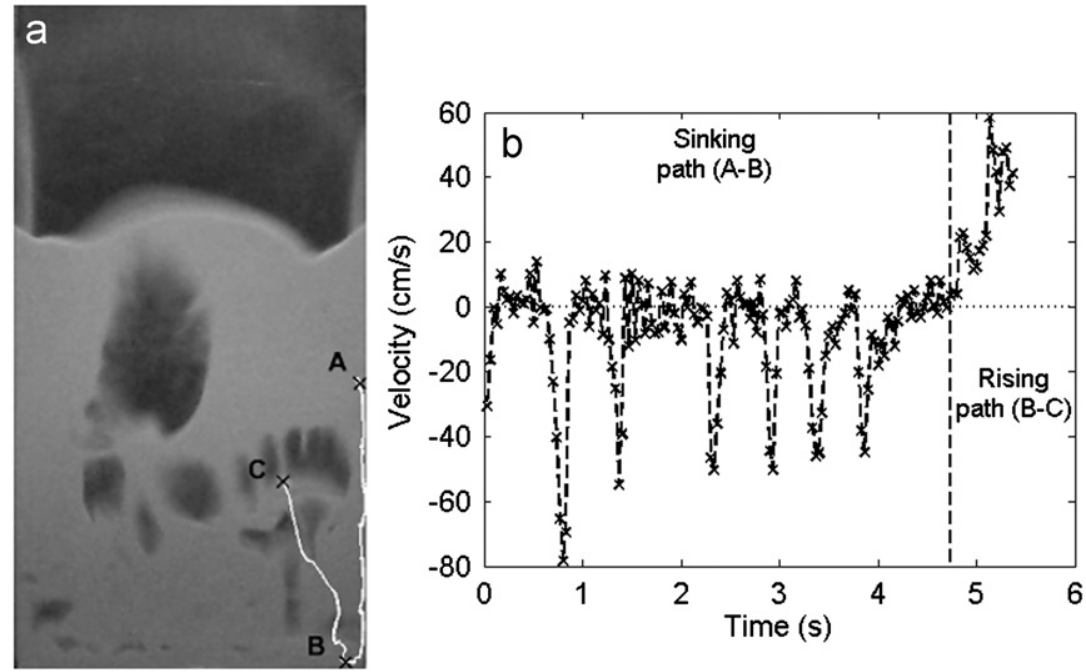

Fig. 7. (a) Object path in the bed and (b) object velocity during the path. Nominal case $\left(U / U_{m f} \quad 2.5\right)$

dimensionless gas velocities (2, 2.5, and 3$)$, the distribution seems rather homogeneous. An increase of the dimensionless gas velocity diminishes the relative importance of buoyant forces, giving a more homogeneous distribution. However for very low dimensionless gas velocities $\left(U / U_{m f}=1.5\right)$, buoyancy effects become important and the object was more probably found in the upper part of the bed.

\subsection{Circulation time}

The circulation time of an object is defined as the time interval between the instants when an object sinks into the bed from its surface until it returns to the surface (object cycle). This is one of the main parameters used to describe the motion of an object in a fluidized bed. It can be compared to the characteristic time of a chemical reaction, when the object considered is a fuel or a catalyst (Gomez Barea and Leckner, 2010). Nevertheless, note that during our experiments the object remained consistent in both size and density, and thus our results cannot be directly extended to processes where the object shrinks or changes its density.

The circulation time of an object will depend directly on its velocity during the cycle, so the mean object velocity was char acterized during both the sinking and the rising paths. Object velocity was determined by the difference in its position in two consecutive images divided by the time interval between images.
In a first approximation, the mean rising and sinking velocities of an object can be determined averaging the measured positive (upwards) and negative (downwards) velocities, respectively; considering that all the positive velocities are grouped in the rising paths, and all the negative velocities occur during the sinking paths. However, the motion of the object is more complex and this simple procedure can lead to important miscalculations of the mean ascending and descending velocities.

Direct observation showed that the motion of an object could be described as a composition of several processes. There was a succession of jumps interleaved with periods, when the object was sinking. During each jump, the object rose in a monotonic ascending manner as it was partially attached to an up coming bubble (or in its trail). The sinking process was more complex. It followed a step like descending motion, characterized by large downwards velocities that were maintained a fraction of a second and separated by low velocity periods, in which the object vibrated over a given position. The word vibration is used, since such a process added up to no net motion in any direction.

These three types of motion can be clearly observed in Fig. 7, where the actual trajectory and velocity of an object in a six second lapse is depicted. Fig. 7a shows an image of the bubbling fluidized bed with the object trajectory superimposed. It consists of a sinking path (from A to $B$ ) and a rising path (from $B$ to $C$ ). The velocities of the object along its trajectory are plotted in Fig. 7b. As stated, the object velocity was always positive (upwards) in the rising path, while the sinking path 
was characterized by small periods (around $0.1 \mathrm{~s}$, with a frequency of around $2 \mathrm{~Hz}$, in Fig. 7b), where the object fell fast, with high negative velocities (downwards), and periods of vibration, in which both negative and positive velocities were present.

Therefore, the rising process can be characterized by the mean ascending velocity inside a jump and the number of jumps, while the sinking process is quite more complex. If we consider it as a whole (for example, from A to B in Fig. 7a), we can calculate a mean descending velocity defined as the distance between $A$ and $B$ divided by the time spent by the object moving from one to the other. This mean velocity will characterize the global sinking motion, even though the object seldom moved at such speed. In contrast, the object moved in the step like manner previously described, alternating large velocities with periods of vibration, in which small ascending and descending velocities occurred.

The mechanisms by which such motion was produced are not evident. It is clear that the source of any net movement is the bubble motion. This is evident enough during the object jumps. The large descending velocities in the step like sinking processes can also be associated with passing bubbles, which were raising dense phase from the bottom and carrying it to the surface of the bed (i.e., carrying particles from below to above the object). Concerning vibration, it can be related with pressure fluctuations in the fluidized bed (also linked with the bubble motion), but further work is needed to clarify this reasoning.

In view of these facts, a time averaged velocity of an object during the sinking path was calculated as a first step to obtain the circulation time. The position of an object was sampled at different frequencies to determine its velocity. Considering the path described by the object in Fig. 7a, we were interested in sampling the position signal at such a frequency that only the object position between instants A and B were considered. Thus, the averaged velocity would be the difference in position at these two instants divided by the time spent by the object moving from $A$ to B. Two complicating factors appeared when doing this automatically: (i) to accurately define point B, so no information of the B C path was used on the mean and (ii) to keep the positive or zero velocities during vibration in the averaging process, since they were part of the sinking process and neglecting them will increase artificially the mean value. The first factor could be minimized by increasing the acquisition frequency, while the second could be minimized by lowering it. Therefore, the sampling frequency had to be defined as a compromise of these two competing factors. Fig. 8 plots the mean sinking velocity calculated for different sampling frequencies, averaging all of the downward velocities in the bed. The mean sinking velocity diminished greatly when the sampling frequency was reduced from 30 to $23 \mathrm{~Hz}$, because vibration was

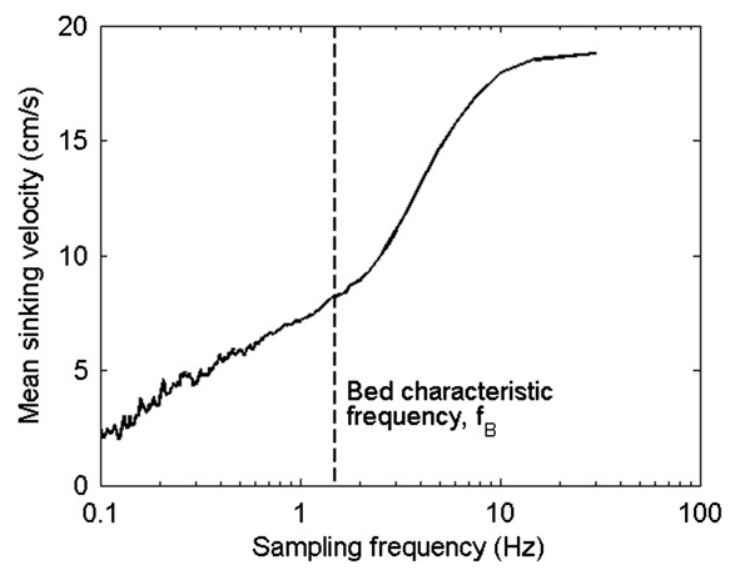

Fig. 8. Mean sinking velocity in the whole bed, sampling the velocity signal at different frequencies.

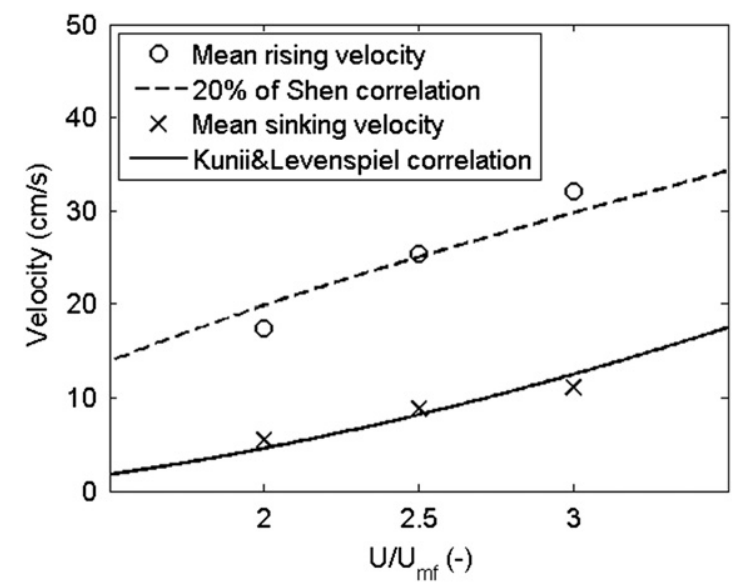

Fig. 9. Mean sinking and rising velocities of an object compared with models for the dense phase and bubbles velocities.

being considered. This frequency $(23 \mathrm{~Hz}$ ) was almost the mea sured characteristic frequency of the bed $(1.51 \mathrm{~Hz})$. The sampling of the signal at lower frequencies produced an exponential decay of the mean sinking velocity, due to the loss of a well established point B. An extremely low sampling frequency would produce the same effect as considering the object going directly from $\mathrm{A}$ to $\mathrm{C}$, describing just a sinking path, but for the total time required. As sampling frequency continues diminishing, the mean sinking velocities would tend to zero. A similar effect was observed for different dimensionless gas velocities. Therefore, the mean sinking velocities were calculated in the following, using a sampling frequency of $2 \mathrm{~Hz}$.

Results of the mean sinking and rising velocities of an object for three different dimensionless gas velocities are presented in Fig. 9. The mean sinking velocity is similar to the Kunii and Levenspiel (1991) correlation for the dense phase downward velocity (Eq. (2)), and it can thus be assumed that the object moves downward with the dense phase, as stated previously in the literature. However, the mean rising velocity represents roughly $20 \%$ of the mean velocity of bubbles, calculated with the widely used correlation of Shen et al. (2004) for the bubble diameter and the correlation of Davidson and Harrison (1963) for the bubble velocity (Eq. (3)). This corresponds well with previous experimental evidence presented in Section 1, although results are widely dispersed in the literature.

$v_{d p}=\frac{f_{w} \delta U_{B}}{1 \delta f_{w} \delta}$

$D_{B}=\left[\left(\frac{8\left(2^{3 / 4} 1\right)}{\lambda}\right)\left(\begin{array}{ll}U & U_{m f}\end{array}\right)\left(h+\frac{\lambda}{\pi\left(2^{3 / 4} 1\right)} \frac{A_{0}}{T}\right)\right]^{2 / 3} g^{-1 / 3}$

$U_{B}=U \quad U_{m f}+\phi \sqrt{ } g D_{b}$

The experimental circulation times obtained for the object throughout the experiments (more than 1200 cycles) are shown in Fig. 10a as a function of the maximum depth attained by an object. It can be observed that the greater the depth, the larger the time periods. The minima follow a linear trend that might be attributed to constant sinking and rising velocities throughout the bed. However, there is considerable scatter in the data. This is due to the incidence of multiple jumps during the rising process associated with a series of passing bubbles that do not carry the object directly to the surface of the bed. Fig. 10b shows the same data arranged as a function of the number of jumps needed to raise the object to the surface of the bed in each cycle. Note that the scattered data for a low number of jumps $(<10 \mathrm{~s})$ fits well with the variation of minima in the time versus depth graph, and thus, 
a

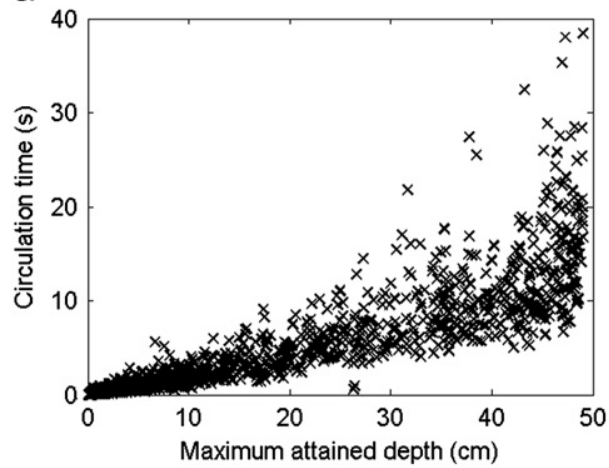

b

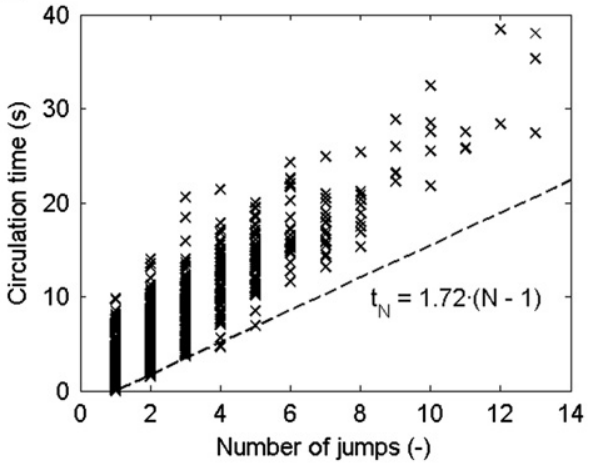

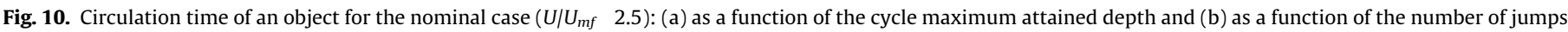
employed to reach the surface of the bed.

a

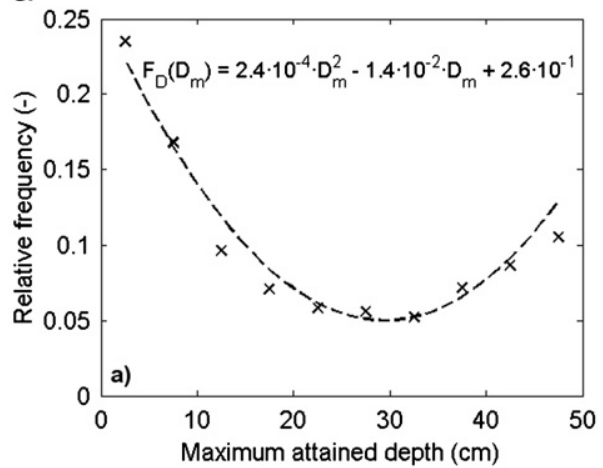

b

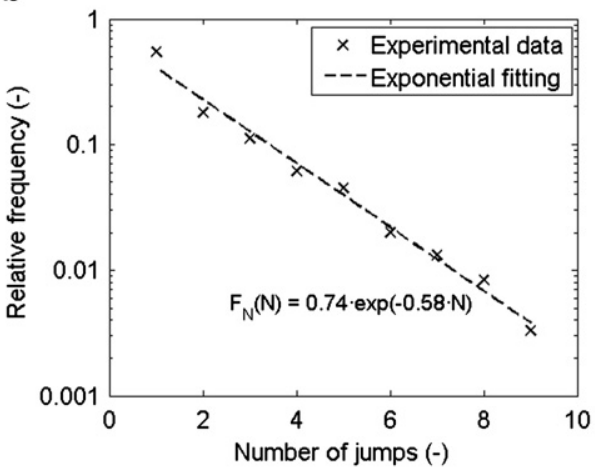

Fig. 11. Relative frequency to (a) reach a determined maximum depth and (b) find cycles with a determined number of jumps. Nominal case $\left(U / U_{m f} \quad 2.5\right)$.

the effects of the number of jumps and the attained depth seem to be cumulative. Of course, a high number of jumps in the rising process are more prone to occur when a greater depth is attained or vice versa. The dashed line, which roughly represents a minimum, was determined by a linear regression of the minimum time for the more probable numbers of jumps (from 1 to 5 ).

The effect of both parameters was studied in more detail. In Fig. 11a, the relative frequency of an object attaining a determined depth is plotted (dividing the bed in slices of $0.05 \mathrm{~m}$ ). This relative frequency was found to follow a parabolic law, with minimum relative frequency at medium depths. This rather surprising result is in accordance with the dense phase behavior, described in Fig. 3b. The object sinks at the sides of the bed (see Fig. 5), and once in such a zone, it is less prone to move sideward to the preferred bubble path (the middle of the bed) than continuing sinking, so the relative frequency to reach a greater depth is higher. Fig. 11b shows the relative frequency of number of jumps that occurs in each cycle. The graph illustrates an exponential decay of the relative frequency with number of jumps. It can be observed that the fitting slightly overestimates the relative frequency to reach high depths and underestimates the relative frequency to find a one jump cycle.

The graphs for the other dimensionless gas velocities that produce a proper circulation of the object $\left(U / U_{m f} 2\right.$ and 3$)$ showed similar results, and are therefore not included here.

\section{Discussion}

An estimate of the circulation time of an object in a bubbling fluidized bed is important when designing fluidized bed applica tions, such as combustors, gasifiers, dryers, or coating reactors.
During our experiments, the circulation time of an object was found to depend mainly on the number of bubbles needed to raise it to the surface of the bed (number of jumps) and the maximum depth attained by the object. Therefore, an estimate of the circulation time for a determined number of jumps $(N)$ and a maximum attained depth $\left(D_{m}\right)$ was made. The estimation, based on semi empirical expressions, consists of two different times: (i) the time due to the number of jumps, $t_{N}$, calculated using the equation in Fig. $10 \mathrm{~b}$ and (ii) the time due to the maximum attained depth, $t_{D}$, calculated assuming a constant velocity for the object. The mean velocities are presented in Fig. 9, both in the sinking $\left(v_{s}\right)$ and the rising $\left(v_{r}\right)$ path.

$$
\begin{aligned}
& t_{N}=1.72\left(\begin{array}{ll}
N & 1
\end{array}\right) \\
& t_{D}=D_{m}\left(\frac{1}{v_{s}}+\frac{1}{v_{r}}\right)
\end{aligned}
$$

The total circulation time $(t)$ can then be calculated as the sum of these two times

$t=1.72\left(\begin{array}{ll}N & 1\end{array}\right)+D_{m}\left(\frac{1}{v_{s}}+\frac{1}{v_{r}}\right)$

The distribution of relative frequencies of the circulation time can also be estimated, using the relative frequencies, to find a determined number of jumps $\left(F_{N}\right)$ and to attain a certain depth $\left(F_{D}\right)$, as shown in Fig. 11. These relative frequencies were transformed into functions of the circulation time, using Eq. (4)

$$
\begin{aligned}
& F_{N}(t)=0.41 e^{-0.34 t} \\
& F_{D}(t)=2.410^{-4} t^{2}\left(v_{s}^{-1}+v_{r}^{-1}\right)^{-2} \quad 1.410^{-2} t\left(v_{s}^{-1}+v_{r}^{-1}\right)^{-1}+2.610^{-1}
\end{aligned}
$$


Finally, the relative frequencies were normalized into prob ability density functions

$P_{N}(t)=0.34 e^{-0.34 t}$

$P_{D}(t)=3.810^{-4} t^{2}\left(v_{s}^{-1}+v_{r}^{-1}\right)^{-2} 2.210^{-2} t\left(v_{s}^{-1}+v_{r}^{-1}\right)^{-1}+0.41$

The functions described in Eq. (7) are the probability density functions of two independent times. The total time spent by the object in a cycle is the sum of these times, as described in Eq. (5). The probability density function for such a sum is the convolution of the two functions

$P_{T}(t)=P_{N}(t) * P_{D}(t)=\int_{0}^{\infty} P_{N}(x) P_{D}(t \quad x) d x$

The probability density function of the total circulation time of the object is plotted in Fig. 12 for the different dimensionless gas velocities. All of the distributions are bi modal due to the shape of an $F_{D}$ (see Fig. 11), which presented a minimum probability for the object to reach moderate depths. Nevertheless, the bi modal shape should not be considered as a sound conclusion, since it was sharpened by errors in the exponential fit of Fig. 11b. As stated previously, the actual probability for $N=1$ is larger than that on the fit. Therefore, if a perfect fit had been used, the first peak would have been larger and the second peak smaller. However, the present, simpler procedure was used for clarity purposes. This procedure gives a proper idea of the global distribution.

The reduction of the dimensionless gas velocity produces an increase in the circulation time as a result of the reduction of both rising and sinking velocities of the object. The rest of the para meters were constant. The probability density functions fitted for the dimensionless gas velocity of 2.5 (Eq. (7)) were used to estimate

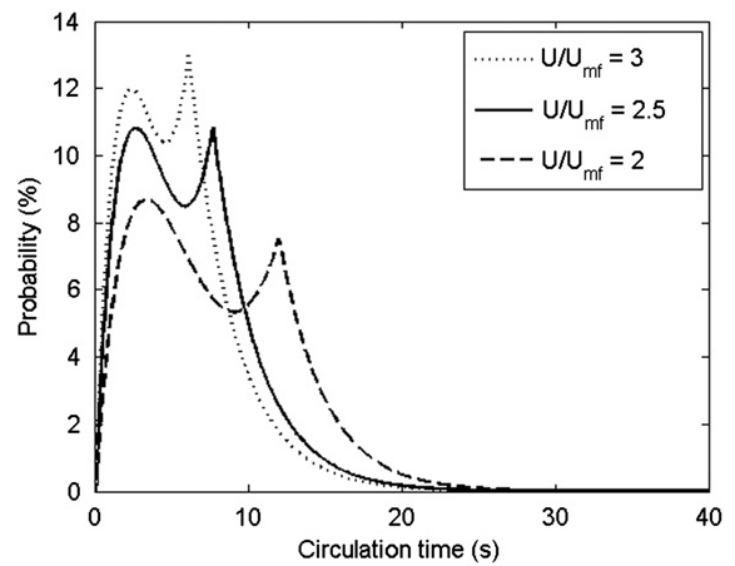

Fig. 12. Probability density function of the total circulation time of an object. the circulation time of the object for the three different dimensionless gas velocities (2, 2.5, and 3 ).

Fig. 13 shows a comparison of our estimate of the circulation time along with the experimental data. The distribution of the experimental circulation times is plotted in a box plot in Fig. 13a for varying dimensionless gas velocities. In a box plot, the distribution of the data is reduced to the median, the lower and upper quartiles (representing $25 \%$ and $75 \%$ of the population), a confidence interval (defined by the two experimental data that are further away from the quartiles, but inside a maximum interval of \pm 1.5 times the inter quartile range), and outliers (that lie out of such a confidence interval). It can be observed that the circulation time decreases with the dimensionless gas velocity, due to the larger sinking and rising velocities of the object. The box plot of the estimated circulation time is also plotted in Fig. 13b. The cross in this diagram represents a circulation time with a probability of $0.1 \%$, which is roughly the occurrence probability of an outlier.

The semi empirical estimation was found to overestimate the lower times (mainly the lower quartile). This overestimation was due to the underestimation of the probability of a single jump to occur in the fitting, as shown in Fig. 11b. The estimation also presents a higher symmetry between the lower and the upper quartiles than the experimental data, due to the overestimation of the probability to attain a high depth (see Fig. 11a). Nevertheless, it seems to accurately predict most of the distribution for the three dimensionless gas velocities. Therefore, the semi empirical expressions used to estimate the circulation time can be said to be in good agreement with the experimental data.

\section{Conclusions}

The motion of a large object within a $2 \mathrm{D}$ bubbling fluidized bed was studied using imaging techniques and considering the circula tion patterns, the object sinking and rising velocities, and the multiple jumps behavior on the rising path. The effect of the dimensionless gas velocity on the main parameters of the motion was also analyzed.

The object employed was larger than the dense phase with a similar density to the bed, although slightly lower. The object circulated throughout the bed following a typical 2 vortex config uration. Its spatial distribution was quite homogeneous when operating with dimensionless gas velocities from 2 to 3, becoming rather non homogeneous (the object remained in the upper zone of the bed most of the time) for low dimensionless gas velocities $\left(U / U_{m f}=1.5\right)$, for which buoyancy effects became important.

The sinking and rising paths were identified, differentiated, and independently characterized. The sinking path of the object was a

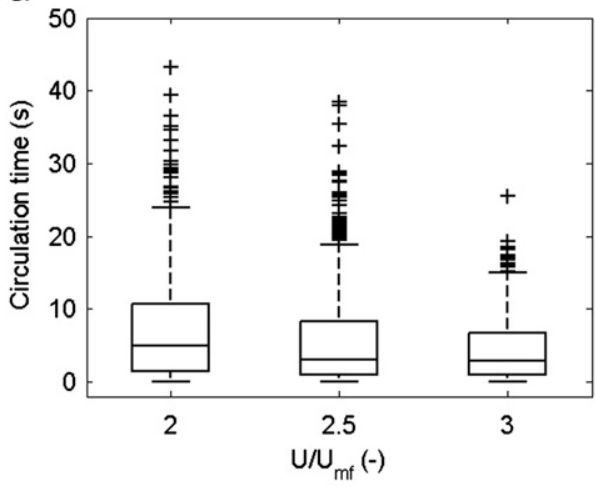

b

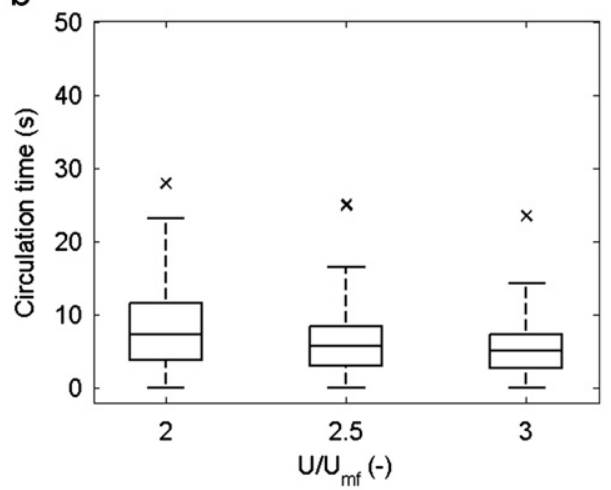

Fig. 13. Box plots of (a) the experimental circulation time and (b) the estimated circulation time. 
characterized by sudden falls with high downwards velocities and vibrations, in which both downwards and upwards velocities were present. However during the rising path (within a single jump), there were only upwards velocities. A mean sinking and rising velocities of an object in the bed was determined considering these facts. The sinking velocity was in accordance with the well known correlation of Kunii and Levenspiel, and thus the effect of buoyant forces on the sinking motion of the object can be considered negligible. The rising velocity was found to be roughly $20 \%$ of the bubbles velocity, a result consistent with previous estimations.

The circulation time of an object depends on the maximum depth attained by the object, and the number of jumps needed for the object to rise back to the surface of the bed. The relation between the circulation time and these two parameters is almost linear. These parameters were considered independent contribu tions to the circulation time, and their probability density functions were obtained. The probability of finding cycles with a determined number of jumps has an inverse exponential relation to the number of jumps, while the probability of attaining a determined depth was found to be parabolic, with a minimum at moderate depths due to the tendency of an object to sink deep before moving sideward to the preferential path of bubbles, typical of the 2 vortex configuration.

Finally, semi empirical expressions were proposed to estimate the circulation time of the object and its probability distribution. The estimate relies on experimental data that are considered independent of the dimensionless gas velocity (provided that the buoyant forces are negligible) and on the mean sinking and rising velocities of the object (that were experimentally obtained for each dimensionless gas velocity, but are in good agreement with widely used correlations). Even though our correlation overestimates the lower times, it was found to accurately predict the majority of the experimental distribution.

\section{Nomenclature}

$A_{0} \quad$ area of the distributor per number of orifices

$C$ relative frequency to find dense phase at a particular location in the bed

$d_{0} \quad$ diameter of the holes of the distributor

$d_{1} \quad$ separation between consecutive holes of the distributor

$D_{m} \quad$ maximum depth attained by the object

$D_{B} \quad$ bubble diameter

$f_{B} \quad$ characteristic frequency of the bed

$f_{w} \quad$ bubble wake fraction

$F_{D} \quad$ relative frequency to attain a certain depth

$F_{N} \quad$ relative frequency of a certain number of jumps

$F_{R} \quad$ relative frequency

$F_{u z} \quad$ relative frequency to find the object at the upper zone of the bed

g gravity

$h \quad$ height measured from the distributor

$h_{B} \quad$ fixed bed height

$H \quad$ vessel height

$N$ number of jumps needed for the object to rise to the surface of the bed

$N_{P} \quad$ total number of images employed in the study of dense phase and bubbles

$P_{D} \quad$ probability density function to attain a certain depth

$P_{N} \quad$ probability density function of the number of jumps

$P_{T} \quad$ probability density function of the circulation time

$P_{N}(t)^{*} P_{D}(t)$ convolution function symbol

$s$ separation between the two lines of holes of the distributor $t \quad$ circulation time

$t_{D} \quad$ minimum circulation time related to the attained depth

$t_{N} \quad$ minimum circulation time related to the number of jump

$T \quad$ bed thickness

$U \quad$ gas velocity

$U_{B} \quad$ bubble velocity

$U_{m f} \quad$ minimum fluidization velocity

$v_{d p} \quad$ downward velocity of the dense phase

$v_{r} \quad$ object mean rising velocity

$v_{s} \quad$ object mean sinking velocity

$W \quad$ bed width

$\Delta P_{B} \quad$ bed pressure drop

$\Delta P_{D} \quad$ distributor pressure drop

$\delta \quad$ bubble fraction in the bed

$\lambda$ constant determined experimentally

$\phi \quad$ constant determined experimentally

\section{Acknowledgments}

This work has been partially supported by the National Energy Program of the Spanish Department of Science and Education (ENE2006 01401) and the Madrid Community (CCG07 uc3m/amb 3412 and CCG08 uc3m/amb 4227).

\section{References}

Almendros-Ibáñez, J.A., Sanchez-Delgado, S., Sobrino, C., Santana, D., 2009. Experimental observations on the different mechanisms for solid ejection in gasfluidized beds. Chemical Engineering and Processing 48-3, 734-744.

Bokkers, G.A., van Sint Annaland, M., Kuipers, J.A.M., 2004. Mixing and segregation in a bidisperse gas-solid fluidised bed: a numerical and experimental study. Powder Technology 140 (3), 176-186.

Davidson, J.F., Harrison, D., 1963. Fluidised Particles first ed. Cambridge University Press, Cambridge.

Du, B., Wei, F., Warsito, W., 2002. Gas and solids mixing in a turbulent fluidized bed. AIChE Journal 48 (9), 1896-1909.

Geldart, D., 1973. Types of gas fluidization. Powder Technology 7, 285-292.

Gomez-Barea, A., Leckner, B., 2010. Modelling of biomass gasification in fluidized bed. Progress in Energy and Combustion Science 36 (4), 444-509.

Grassler, T., Wirth, K.-E., 2000. X-ray computer tomography-potential and limitation for the measurement of local solids distribution in circulating fluidized beds. Chemical Engineering Journal 77 (1-2), 65-72.

Hoffmann, A.C., Janssen, L.P., Prins, J., 1993. Particle segregation in fluidized binary mixtures. Chemical Engineering Science 48 (9), 1583-1592.

Ibsen, C.H., Solberg, T., Hjertager, B.H., Johnsson, F., 2002. Laser Doppler anemometry measurements in a circulating fluidized bed of metal particles. Experimenta Thermal and Fluid Science 26 (6-7), 851-859.

Johnsson, F., Zijerveld, R.C., Schouten, J.C., van den Bleek, C.M., Leckner, B., 2000. Characterization of fluidization regimes by time-series analysis of pressure fluctuations. International Journal of Multiphase Flow 26, 663-715.

Karri, S.B.R., Werther, J., 2003. Gas distributor and plenum design in fluidized beds. In: Yang, W.C. (Ed.), Handbook of Fluidization and Fluid-particle Systems. Marcel Dekker Inc., New York, pp. 164-179.

Koornneef, J., Junginger, M., Faaij, A., 2007. Development of fluidized bed combustion-an overview of trends, performance and cost. Progress in Energy and Combustion Science 33, 19-55.

Kunii, D., Levenspiel, O., 1991. Fluidization Engineering second ed. ButterworthHeinemann, Boston.

Laverman, J.A., Roghair, I., van Sint Annaland, M., Kuipers, H., 2008. Investigations into the hydrodynamics of gas-solid fluidized beds using particle image velocimetry coupled with digital image analysis. The Canadian Journal of Chemical Engineering 86, 523-535.

Lim, K.S., Agarwal, P.K., 1994. Circulatory motion of a large and lighter sphere in a bubbling fluidized bed of smaller and heavier particles. Chemical Engineering Science 49 (3), 421-424.

Müller, C.R., Davidson, J.F., Dennis, J.S., Hayhurst, A.N., 2007. A study of the motion and eruption of a bubble at the surface of a two-dimensional fluidized bed using particle image velocimetry (PIV). Industrial and Engineering Chemical Research 46, 1642-1652.

Nienow, A.W., Rowe, P.N., Chiba, T., 1978. Mixing and segregation of a small portion of large particles in gas fluidized beds of considerably smaller ones. AIChe Symposium Series 74, 45-53.

Nguyen, T.H., Grace, J.R., 1978. Forces on objects immersed in fluidized beds. Powder Technology 19, 255-264.

Otsu, N., 1979. A threshold selection method from gray-level histograms. IEEE Transactions on Systems, Man, and Cybernetics 9, 62-66. 
Pallarès, D., Johnsson, F., 2006. A novel technique for particle tracking in cold 2-dimensional fluidized beds-simulating fuel dispersion. Chemical Engineering Science 61, 2710-2720.

Rees, A.C., Davidson, J.F., Hayhurst, A.N., 2005. The rise of a buoyant sphere in a gasfluidized bed. Chemical Engineering Science 60, 1143-1153.

Rios, G.M., Dang Tran, K., Masson, H., 1986. Free object motion in a gas fluidized bed Chemical Engineering Communications 47, 247-272.

Rowe, P.N., 1973. Estimation of solid circulation rate in bubbling fluidised bed Chemical Engineering Science 28, 979-980.
Sanchez-Delgado, S., Marugan-Cruz, C., Acosta-Iborra, A., Santana, D., 2010. Dense phase fluctuation in a 2-D fluidized bed. Powder Technology 200, 37-45.

Shen, L., Johnsson, F., Leckner, B., 2004. Digital image analysis of hydrodynamics two-dimensional bubbling fluidized beds. Chemical Engineering Science 59, 2607-2617.

Stein, M., Ding, Y.L., Seville, J.P.K., Parker, D.J., 2000. Solids motion in bubbling gas fluidised beds. Chemical Engineering Science 55, 5291-5300.

Sveen, J.P. 〈http://www.math.uio.no/\%7E\%7b\%7djks/matpiv〉 (Last modified in August, 2004) 